\title{
Simulation of the Performance of a Fundamental Neutron Physics Beamline at the High Flux Isotope Reactor
}

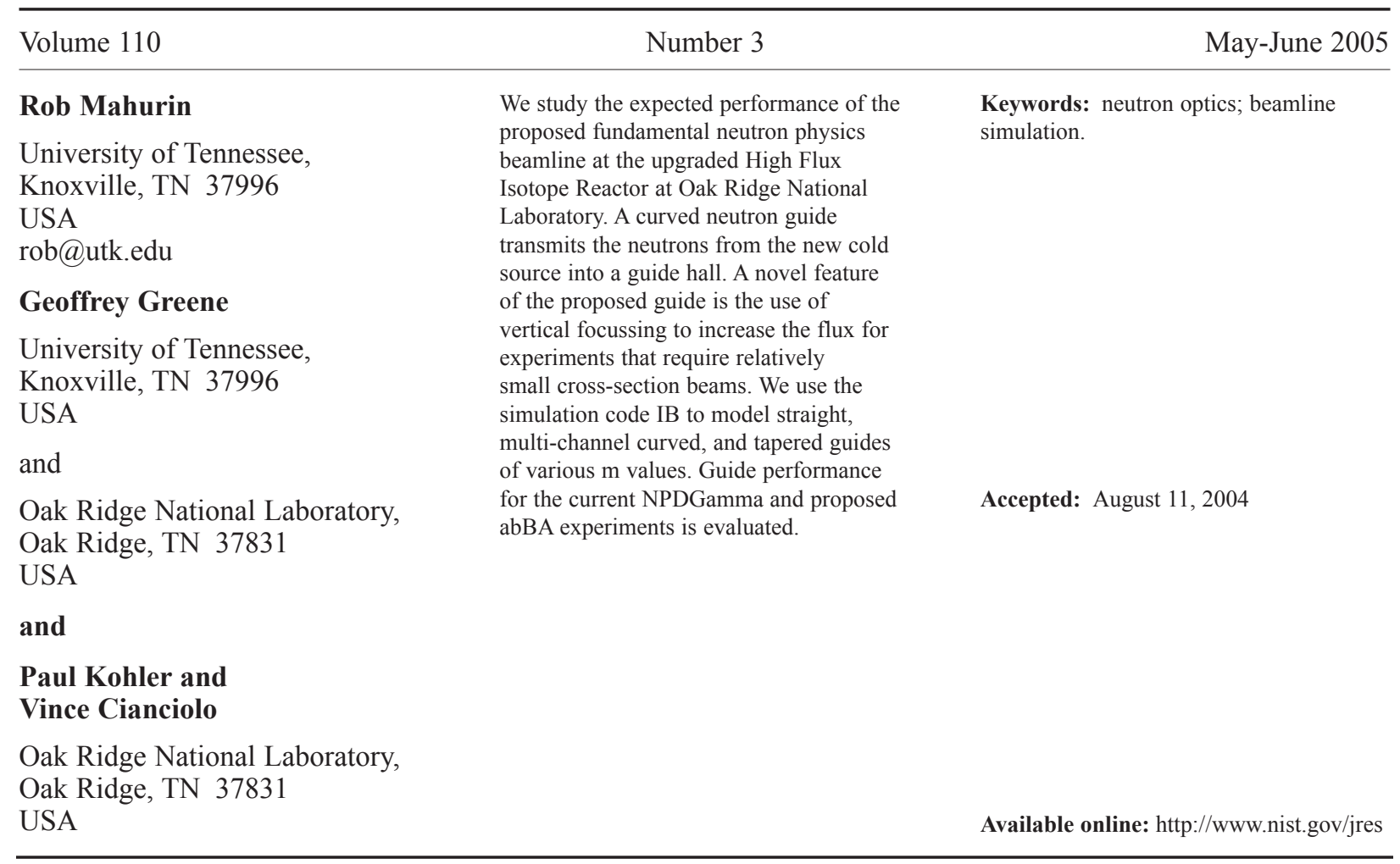

\section{Neutron Physics at HFIR}

The High Flux Isotope Reactor [1] at Oak Ridge National Laboratory is an 85 MW "flux-trap" reactor designed for the production of transuranium isotopes. The reactor first came on-line in 1966, underwent an upgrade in the late $1980 \mathrm{~s}$, and is presently undergoing a second refurbishment; during running periods the reactor is typically active for $\approx 5000$ hours a year. When this upgrade is complete HFIR will be comparable to the most intense continuous source of cold neutrons in the world. The HFIR refurbishment includes the construction of a new guide hall, which will contain the fundamental physics beamline modelled here.

In this paper we evaluate the simulated guides based on their expected performance for the abBA [2] and NPDGamma [3] experiments. The figure of merit used for the abBA experiment is the neutron capture flux $\left(\mathrm{n} / \mathrm{cm}^{3}\right)$ in a small volume located $\approx 2.5 \mathrm{~m}$ from the end of the guide. For the NPDGamma experiment the figure of merit is the neutron fluence $(\mathrm{n} / \mathrm{s})$ in a circular aperture $\approx 10 \mathrm{~cm}$ in diameter located $2 \mathrm{~m}$ from the end of the guide.

The reader should note that these simulations only deal with the behavior of the guide, and do not account for losses due to windows and other effects. 


\section{The Cold Source and Upstream Guide}

The cold moderator at HFIR is a square $8.52 \mathrm{~cm}$ on a side whose spectrum is shown in Fig. 1. The CG4 guide, which begins $5.297 \mathrm{~m}$ from the cold source, is a $15 \mathrm{~m}$ long, $1.9 \mathrm{~cm}$ wide, 4 channel bender with radius $107.43 \mathrm{~m}$ and supermirror parameter $m=2.5$, followed by a $10 \mathrm{~m}$ long, $m=2$ straight section. The bender tapers from $11.56 \mathrm{~cm}$ to $15 \mathrm{~cm}$ tall over the first $6 \mathrm{~m}$ of its length. At this point there is a gap for a triple-axis spectrometer, followed by about $10 \mathrm{~m}$ of guide leading to the fundamental physics experiments. Only the guide parameters of the section after this gap were varied.

The design calculations of the cold source and the CG4 guide [4] predict a value for the flux at $30 \mathrm{~m}$ of $1.97 \times 10^{9} \mathrm{n} / \mathrm{s} / \mathrm{cm}^{2}$. The simulations described here give a flux $1.90 \times 10^{9} \mathrm{n} / \mathrm{s} / \mathrm{cm}^{2}$, an agreement within about 5 $\%$.

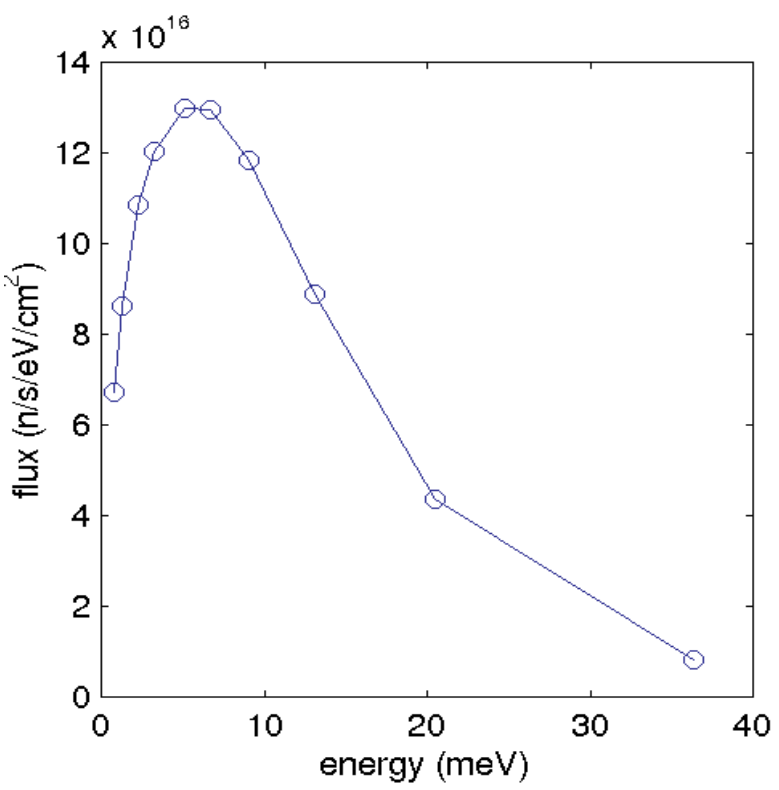

Fig. 1. The spectrum of the cold moderator [4]. The integrated flux is $2.2 \times 10^{15} \mathrm{n} / \mathrm{s} / \mathrm{cm}^{2}$.

\section{The Fundamental Neutron Physics Beamline}

Extending the guide poses two challenges. One is space: the CG4 guide is nearly parallel to the adjacent beamline, and a straight extension would not leave adequate room for experiments. This problem is addressed by having a bender in the extension. The second problem is that the CG4 guide has an unfavorable aspect ratio $\left(1.9 \times 15 \mathrm{~cm}^{2}\right)$ for the proposed fundamental physics experiments, which require fairly small cross-section beams. This problem is addressed by the vertical focussing.

The following parameters were varied to maximize the figure-of-merit "signals" described above:

- the entrance size

- the clearance from the adjacent beam line

- the channel width

- the vertical taper.

These degrees of freedom are largely independent of each other. The results of these simulations are described here.

\subsection{Entrance Size}

Figure 2 shows the neutron intensity at the entrance to the fundamental physics beamline, and the fraction of the incident beam that enters a different-sized guides. We chose to model guides with $4 \times 15 \mathrm{~cm}^{2}$ entrances, keeping $95 \%$ of the available neutrons.
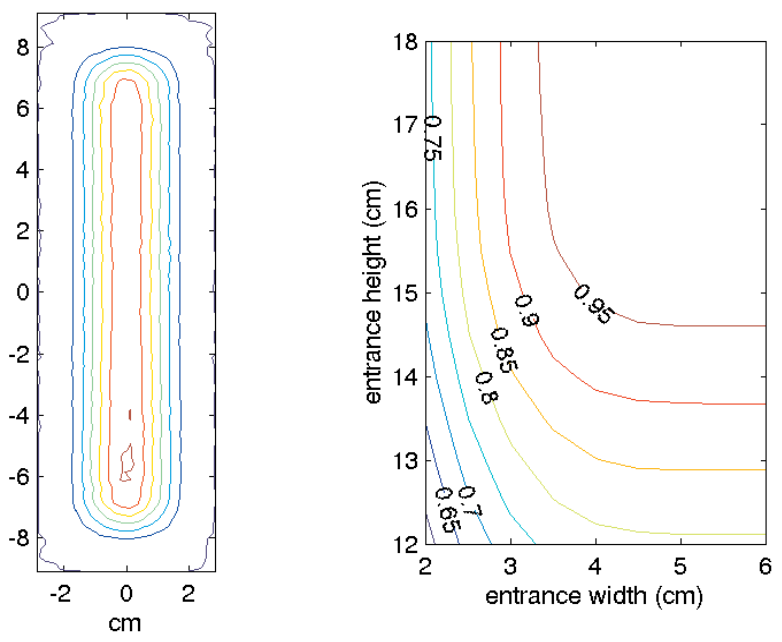

Fig. 2. (a) Beam profile at the entrance to the fundamental physics beamline, $\approx 31.8 \mathrm{~m}$ from the moderator. The increased intensity at the bottom of the beam is due to the neutrons' gravitational fall. (b) Curves in this panel are labeled with the fraction of incident neutrons accepted for the given horizontal and vertical aperture.

\subsection{Clearance From the Adjacent Guide}

A clearance of $\approx 1.1 \mathrm{~m}$ from the undeflected guide path was desirable to have adequate space for the experiments. In this paper we consider only guides that accomplish this using a single bender. Table 1 summarizes the effect of bending the beam to attain this deflection. The guides in this table have the optimum number of channels, described in the next section. 
Table 1. Tradeoff between flux and floor space

\begin{tabular}{ccc}
\hline \hline Clearance $(\mathrm{m})$ & Bender radius $(\mathrm{m})$ & Guide transmission \\
\hline 0 & $\infty$ & 0.96 \\
0.650 & 115.29 & 0.63 \\
0.875 & 85.71 & 0.56 \\
1.100 & 68.18 & 0.49 \\
\hline
\end{tabular}

\subsection{Optimizing Channel Width}

A single-channel bender is characterized by four parameters: the channel width $d$, the radius of curvature $r$, the length $L$, and the supermirror parameter $m$. It is convenient to define a sight angle $\gamma=\sqrt{2 d / r}$, which allows one to consider the guides in a scale-free way [5]. For a $4 \mathrm{~cm}$ wide $m=3.5$ bender illuminated by the HFIR spectrum, the optimum simulated $\gamma \simeq 9.5 \mathrm{mrad}$. The transmission is then roughly the reflectivity raised to the number of bounces along the guide.

For a bender with a very large number of channels, loss on the vanes at the bender entrance becomes important. The universal transmission curve shown in Fig. 3, combined with this entrance loss, is an accurate predictor of the behavior of the guide. For example, a $70 \mathrm{~m}$ radius bender is found by this method to require 10 channels. The optimal number of channels as a function of radius is shown in Fig. 4.
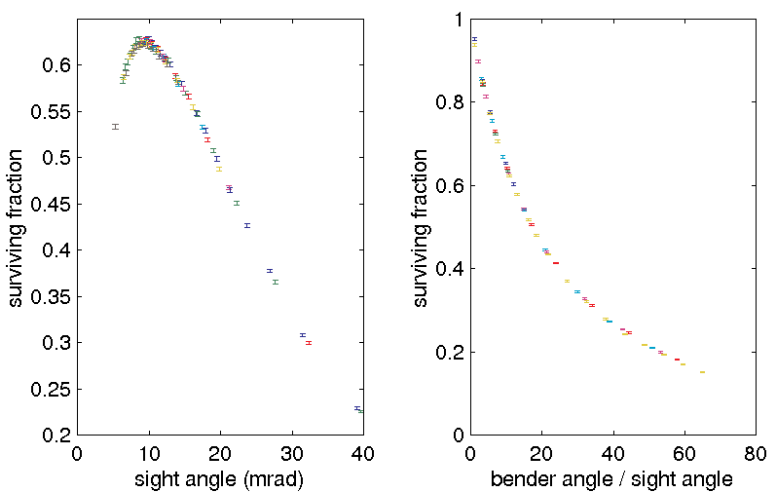

Fig. 3. Universal behavior of benders. (a) Transmission through $100 \mathrm{mrad}$ benders with different sight angles. The optimum sight angle is around $9.5 \mathrm{mrad}$. (b) Transmission through benders with the same sight angle but different lengths. Color in both cases represents benders of different radius; the radius was varied from 5 to $100 \mathrm{~m}$. Uncertainties are statistical.

\subsection{Vertical Tapering}

The CG4 guide was designed for a tall, narrow beam. However, both of the nuclear physics experiments described here require small cross-section neutron beams. Because our source is an $m=2$ guide, we have

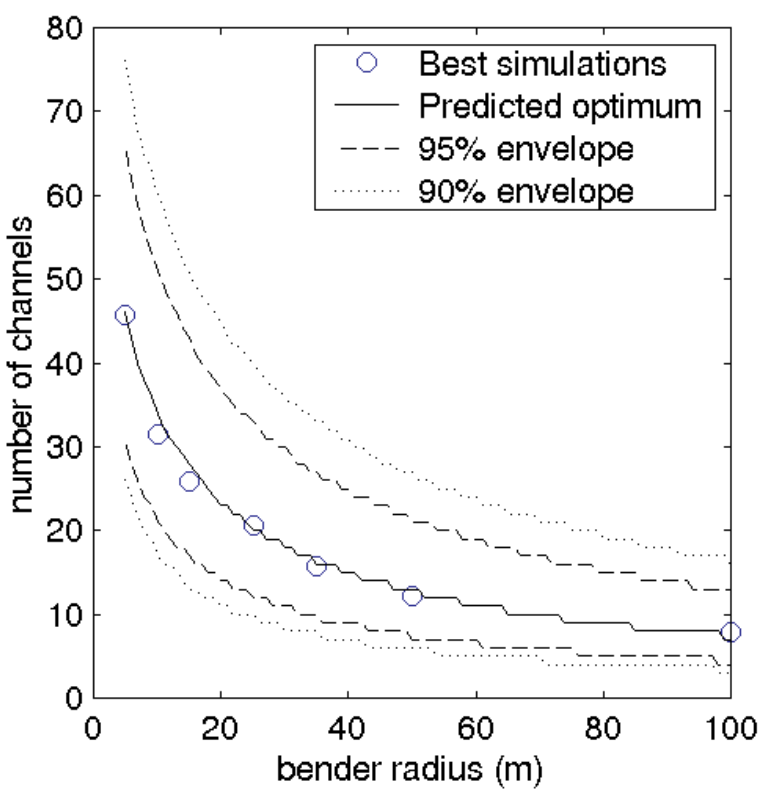

Fig. 4. Optimal numbers of channels for benders of radius $5-100 \mathrm{~m}$ are shown by the solid curve. Benders are $4 \mathrm{~cm}$ wide and bend through $10 \mathrm{mrad}$. The dashed curves show the numbers of channels for which the exit flux is reduced by $5 \%$ and $10 \%$. All the curves were obtained by combining the universal bender response in Fig. 3 with absorption on the vanes at the bender entrance; the circles indicate the best number of channels in a series of simulations at a given radius.

the opportunity to use an $m=3$ or $m=3.5$ guide with a vertical taper to provide spatial compression. The response to this taper is shown in Fig.5.

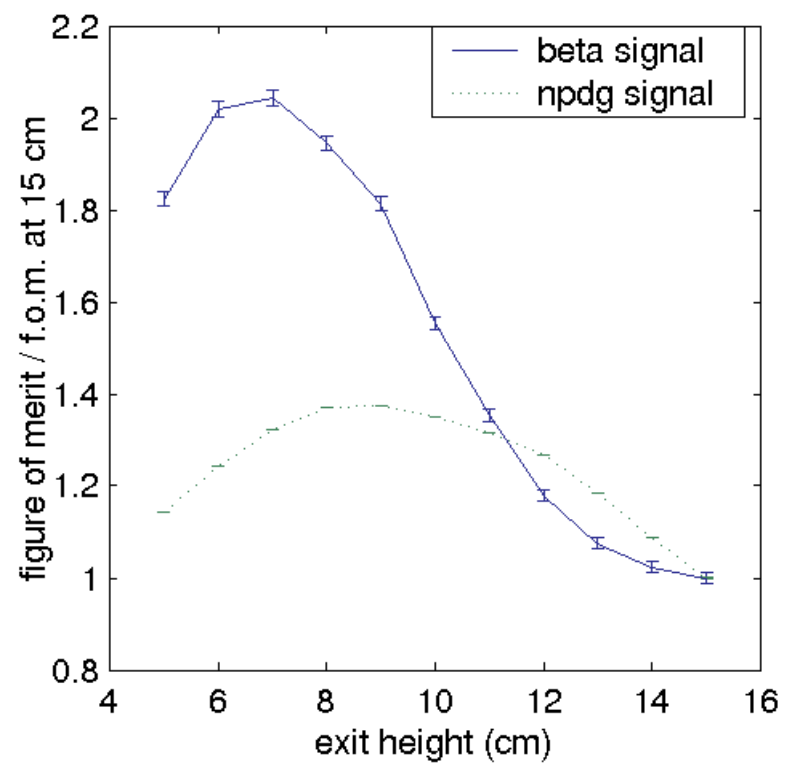

Fig. 5. The effect of a simple vertical taper. See discussion in Sec. 1 for the definition of the figure of merit for the different experiments. Notice that the beta decay signal doubles as the vertical height of the beam is reduced by the taper to $7 \mathrm{~cm}$. The shapes of the curves are independent of the bender radius. Uncertainties are statistical. 


\section{Conclusions}

A curved, converging guide at the High Flux Isotope Reactor provides an attractive facility for the suggested experiments. The optimal guide configurations (with $1.1 \mathrm{~m}$ clearance) provided the following:

- $1 \times 10^{4} \mathrm{n} / \mathrm{cm}^{3}$ in the abBA detector, and

- $3 \times 10^{10} \mathrm{n} / \mathrm{s}$ in the NPDGamma detector (n.b. This is nearly an order of magnitude larger than the total fluence at LANSCE flight path 12).

These calculations deal only with the behavior of the guide and do not account for window losses etc. We note that modest additional gains may be expected by having a short bender followed by a straight tapered section. The use of a polygonal approximation to an elliptical focussing guide, rather than the simple taper investigated here, may improve performance as well.

\section{Acknowledgements}

We are indebted to J. K. Zhao for his assistance with the IB simulation code. This project was supported by the DOE Office of Nuclear Physics (DE-FG0203ER41258) and ORNL.

\section{References}

[1] For more information about the High Flux Isotope Reactor please see the web site http://www.ornl.gov/sci/hfir/.

[2] W. S. Wilburn et al., this Special Issue.

[3] S. A. Page et al., this Special Issue.

[4] Greg Smith, private communication to P. K.

[5] D. Dubbers, Transmission of a lossy curved supermirror neutron guide, Nuclear Instr. Meth. Phys. Res. A 349, 302-306 (1994). 